\title{
Load Shedding in Microgrids with Dual Neural Network and AHP Algorithm
}

\author{
Le Thi Hong Nhung \\ Electrical and Electronics Department \\ HCMC University of Technology and Education \\ Ho Chi Minh City, Vietnam \\ nhunglth@hcmute.edu.vn \\ Hoang Minh Vu Nguyen \\ Urban Engineering Department \\ HCMC University of Architecture \\ Ho Chi Minh City, Vietnam \\ vu.nguyenhoangminh@uah.edu.vn \\ Thai An Nguyen \\ Electrical and Electronics Department \\ Cao Thang Technical College \\ Ho Chi Minh City, Vietnam \\ nguyenthaian@caothang.edu.vn
}

\author{
Trieu Tan Phung \\ Electrical and Electronics Department \\ Cao Thang Technical College \\ Ho Chi Minh City, Vietnam \\ phungtrieutan@caothang.edu.vn
}

Trong Nghia Le

Electrical and Electronics Department

HCMC University of Technology and Education

Ho Chi Minh City, Vietnam

trongnghia@hcmute.edu.vn

Vo Tan Danh

Electrical and Electronics Department

HCMC University of Technology and Education

Ho Chi Minh City, Vietnam

2080602@student.hcmute.edu.vn

\begin{abstract}
This paper proposes a new load shedding method based on the application of a Dual Neural Network (NN). The combination of a Back-Propagation Neural Network (BPNN) and of Particle Swarm Optimization (PSO) aims to quickly predict and propose a load shedding strategy when a fault occurs in the microgrid (MG) system. The PSO algorithm has the ability to search and compare multiple points, so the proposed NN training method helps determine the link weights faster and stronger. As a result, the proposed method saves training time and achieves higher accuracy. The Analytic Hierarchy Process (AHP) algorithm is applied to rank the loads based on their importance factor. The results of the ratings of the loads serve as a basis for constructing the load shedding strategies of a $\mathrm{NN}$ combined with the PSO algorithm (ANN-PSO). The proposed load shedding method is tested on an IEEE 25-bus 8-generator MG power system. The simulation results show that the frequency recovery of the power system is positive. The proposed neural network adapts well to the simulated data of the system and achieves high performance in fault prediction.
\end{abstract}

Keywords-load shedding; ANN-PSO;BPNN; Dual Neural Network; $A H P$

\section{INTRODUCTION}

The increase in load consumption over time poses problems of power system stability. To ensure balance in terms of power, the power system must constantly change in size and structure [1]. However, in some unexpected cases such as serious problems in the power system leading to an imbalance of active power, it is necessary to have temporary solutions to restore the equilibrium of the system. There are many solutions to this problem, as shown in [2], the self-healing ability of the power system depends on the load adjustment based on the voltage, frequency and governor characteristics of the generating sets. In [3-4], traditional load shedding methods are presented using under frequency load shedding relay to set the threshold of the shedding frequency. Currently, many new load shedding methods apply intelligent algorithms. In [5], the decisionmaking technique in load shedding is presented using the AHP algorithm. In [6], the application of the Ant Colony Optimization (ACO) algorithm is presented in optimizing the amount of load shedding power. In [7-8], the PSO algorithm is applied to optimize the inertia weights and train coefficients to get the optimal settings for the system and improve the voltage quality. In [9], a new adaptive load shedding method based on ANNs and proposed shedding power detection is presented. The ANN is used to estimate the total amount of active power that causes unbalance over the attenuation frequency period, equivalent from the rated value to the threshold value. In [10], the Genetic Algorithm (GA) is also applied to optimal shedding against voltage collapse. However, the GA algorithm has the disadvantage of a very long training time compared to PSO algorithm.

The above studies often use intelligent methods and algorithms individually. Therefore, the results obtained are usually satisfactory but not the best because of the limitations of each method and algorithm. The proposed idea is to combine methods together, thereby using the advantages of one method 
to overcome the disadvantages of the other method. In this paper, a new load shedding method is proposed based on the application of a dual NN system structured from a Bayesian NN (BNN) and a NN combined with PSO (ANN-PSO), thereby quickly predicting incidents and recommending load shedding strategies. In the structure of the proposed neural network, the $\mathrm{BNN}$ is responsible for classifying the fault types in the system to make a decision whether or not to shed the load. The NN combines the PSO with the task of determining the load shedding strategies based on the fault type and fault location in the system, so the proposed NN training method can determine the link weights faster and stronger. Tthe training time is saved and the accuracy is high. The AHP algorithm is applied in this paper to rank the loads based on a hierarchical model of comparing their importance to each other. The ranking results of the loads are the basis for constructing the load shedding strategies of the ANN-PSO. The proposed method in the article helps solve the integration problem in the load shedding issue, quickly determining whether or not to shed the load and propose a strategy for shedding the load quickly.

The effectiveness of the proposed method is simulated and tested on the IEEE 25-bus 8-generator MG system. The simulation results show the efficiency of the power system in frequency recovery. The frequency value quickly returns to the allowable range when a fault occurs after the intervention of load shedding according to the proposed method. The proposed $\mathrm{NN}$ adapts well to the simulation data of the IEEE 25-bus 8generator MG system and achieves high performance in fault prediction.

\section{THE LOAD SHEDDING CONTROL STRATEGY CONSTRUCTION BASED ON THE AHP ALGORITHM}

AHP algorithm is a method of weight calculating applied to multi-criteria decision problems with the idea of using knowledge and importance priority to rank objects in a system [11-13]. Here, the AHP algorithm is applied to determine the importance factor and rank load unit in the IEEE 25 bus power system. A hierarchical model of the importance comparison of load centers and loads to each other proposed by a system expert or operator is shown in Figure 1. The steps to implement the AHP algorithm are detailed in [5].

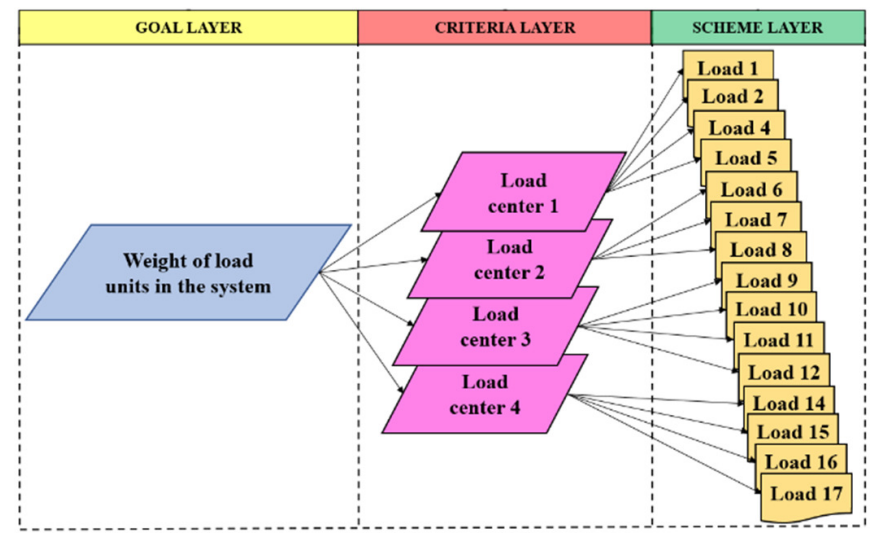

Fig. 1. The AHP hierarchical model includes load centers and load units.
The construction of a judgment matrix of the importance factor of the load centers and of the loads in each load center is presented in Tables I and II. The AHP algorithm is applied to calculate the importance factor of the load centers and the load nodes in each load center. The result and load's rank are presented in Table III.

TABLE I. JUDGMENT MATRIX OF LOAD CENTERS

\begin{tabular}{|l|c|c|c|c|}
\hline & Load center & Load center & Load center & Load center \\
& $\mathbf{1}$ & $\mathbf{2}$ & $\mathbf{3}$ & $\mathbf{4}$ \\
\hline Load center 1 & 1 & 1 & $1 / 3$ & $1 / 5$ \\
\hline Load center 2 & 1 & 1 & $1 / 2$ & $1 / 3$ \\
\hline Load center 3 & 3 & 2 & 1 & $1 / 3$ \\
\hline Load center 4 & 5 & 3 & 3 & 1 \\
\hline
\end{tabular}

TABLE II. JUDGMENT MATRIX OF LOAD UNITS IN THE LOAD CENTERS

\begin{tabular}{|c|c|c|c|c|}
\hline LC $_{\mathbf{1}}$ & Load 1 & Load 2 & Load 4 & Load 5 \\
\hline Load 1 & 1 & 1 & $1 / 3$ & $1 / 2$ \\
\hline Load 2 & 1 & 1 & $1 / 3$ & $1 / 2$ \\
\hline Load 4 & 3 & 3 & 1 & 2 \\
\hline Load 5 & 2 & 2 & $1 / 2$ & 1 \\
\hline LC $_{\mathbf{2}}$ & Load 6 & Load 7 & Load 8 & \\
\hline Load 6 & 1 & $1 / 3$ & $1 / 3$ & \\
\hline Load 7 & 3 & 1 & $1 / 2$ & \\
\hline Load 8 & 3 & 2 & 1 & \\
\hline LC 3 & Load 9 & Load 10 & Load 11 & Load 12 \\
\hline Load 9 & 1 & 2 & 2 & $1 / 2$ \\
\hline Load 10 & $1 / 2$ & 1 & 2 & $1 / 3$ \\
\hline Load 11 & $1 / 2$ & $1 / 2$ & 1 & $1 / 2$ \\
\hline Load 12 & 2 & 3 & 2 & 1 \\
\hline LC 4 & Load 14 & Load 15 & Load 16 & Load 17 \\
\hline Load 14 & 1 & 2 & 2 & 3 \\
\hline Load 15 & $1 / 2$ & 1 & 2 & 3 \\
\hline Load 16 & $1 / 2$ & $1 / 2$ & 1 & 2 \\
\hline Load 17 & $1 / 3$ & $1 / 3$ & $1 / 2$ & 1 \\
\hline
\end{tabular}

TABLE III. RANKING OF LOAD SHEDDING ACCORDING TO THE AHP

\begin{tabular}{|c|c|c|c|c|c|}
\hline Rank & Load & $\begin{array}{c}\text { Load } \\
\text { center }\end{array}$ & $\mathbf{W}_{\mathbf{L j}}$ & $\mathbf{W L}_{\mathbf{C i}}$ & $\begin{array}{c}\text { Weights of the } \\
\text { importance } \\
\text { factor } \mathbf{W}_{\text {ij }}\end{array}$ \\
\hline 1 & Load 1 & $\mathrm{LC}_{1}$ & 0.14114490 & 0.10314687 & 0.014558655 \\
\hline 2 & Load 2 & $\mathrm{LC}_{1}$ & 0.14114490 & 0.10314687 & 0.014558655 \\
\hline 3 & Load 6 & $\mathrm{LC}_{2}$ & 0.13964794 & 0.12970033 & 0.018112384 \\
\hline 4 & Load 5 & $\mathrm{LC}_{1}$ & 0.26270020 & 0.10314687 & 0.027096703 \\
\hline 5 & Load 1 & $\mathrm{LC}_{3}$ & 0.13498819 & 0.24139951 & 0.032586083 \\
\hline 6 & Load 10 & $\mathrm{LC}_{3}$ & 0.17249955 & 0.24139951 & 0.041641307 \\
\hline 7 & Load 7 & $\mathrm{LC}_{2}$ & 0.33251593 & 0.12970033 & 0.043127426 \\
\hline 8 & Load 4 & $\mathrm{LC}_{1}$ & 0.45501010 & 0.10314687 & 0.046932868 \\
\hline 9 & Load 17 & $\mathrm{LC}_{4}$ & 0.10779910 & 0.52575330 & 0.056675733 \\
\hline 10 & Load 9 & $\mathrm{LC}_{3}$ & 0.26997638 & 0.24139951 & 0.065172166 \\
\hline 11 & Load 8 & $\mathrm{LC}_{2}$ & 0.52783613 & 0.12970033 & 0.068460520 \\
\hline 12 & Load 16 & $\mathrm{LC}_{4}$ & 0.18671352 & 0.52575330 & 0.098165249 \\
\hline 13 & Load 12 & $\mathrm{LC}_{3}$ & 0.42253588 & 0.24139951 & 0.101999954 \\
\hline 14 & Load 15 & $\mathrm{LC}_{4}$ & 0.29222244 & 0.52575330 & 0.153636912 \\
\hline 15 & Load 14 & $\mathrm{LC}_{4}$ & 0.41326494 & 0.52575330 & 0.217275406 \\
\hline
\end{tabular}

Based on the results of loads' rank, the load that has a lower importance factor will be prioritized for shedding and vice versa. In detail, based on the results in Table I, the Load 1 will be prioritized for shedding first and Load 14 which has the highest importance factor will be shedding last. The implementation of load shedding is performed when the grid frequency attenuation is less than the allowable value and corresponds to the cases in which the MG operates in the island 
grid separation mode or a generator failure requires implement load shedding. The process of implementing this load shedding strategy is carried out until the frequency recovers to the allowable range from $49.8 \mathrm{~Hz}$ to $50.2 \mathrm{~Hz}$.

\section{BACKPROPAGATION NEURAL NETWORK}

The structure of the ANN network consists of 3 main parts, the input layer, the hidden layer, and the output layer. The signal will be the input of the network and will be processed to give the answer. The number of nodes of the layers and the network structure is chosen to suit each problem and training data. BPNN is a feedforward multilayer network using the back propagation error training algorithm proposed in 1986. It is used in many fields. Thanks to its ability to self-learn from errors, the network structure is very suitable for nonlinear problems. The flowchart of the training process of a BPNN can be seen in [14]. However, it also has some disadvantages such as low convergence speed and instability [15]. In order to solve the limitation BPNN the author in [16] proposed to combine it with the Genetic Algorithm (GA) to improve its structure. However, in GA, chromosomes exchange information with each other through crossover, which is a two-way information sharing mechanism with long loop times. PSO algorithm uses multi-point comparison search, so the proposed training method can determine the link weights faster, saving training time and achieving high accuracy [17].

\section{PARTICLE SWARM OPTIMIZATION ALGORITHM}

The PSO algorithm was proposed in [18]. In the field of power systems, algorithms are often applied in optimization problems [17]. With PSO, each element in the swarm is represented as a vector. In the $\mathrm{NN}$ structure optimization problem, each weight vector represents the ANN structure consisting of the weight values of all the nodes in the neural network. Each particle (element) remembers its individual best historical location, called $\mathrm{P}_{\text {best }}$. For each iteration, the best global position $\mathrm{G}_{\text {best }}$ is found. Once the best global position is found, each element will move closer to its individual best position and best global position. After many iterations, this process finds a good network structure for the objective function, since the elements converge to a quasi-optimal solution. The speed and position of each individual are calculated as:

$$
\begin{aligned}
\mathrm{v}_{\mathrm{i}}^{\mathrm{k}+1}= & \left.\mathrm{w} \cdot \mathrm{v}_{\mathrm{i}}^{\mathrm{k}}+\mathrm{c}_{1} \cdot \operatorname{rand}_{1} \mathrm{O}\right) \cdot\left(\mathrm{p}_{\text {besti }}-\mathrm{x}_{\mathrm{i}}^{\mathrm{k}}\right)+ \\
& \mathrm{c}_{2} \cdot \operatorname{rand}_{2}(\mathrm{O}) \cdot\left(\mathrm{g}_{\text {best }}-\mathrm{x}_{\mathrm{i}}^{\mathrm{k}}\right)
\end{aligned}
$$

After each cycle, the position of each individual will be updated as follows:

$$
x_{i}^{k+1}=x_{i}^{t}+v_{i}^{k+1}
$$

where $\mathrm{w}$ is the inertial weight, $\mathrm{c}_{1}, \mathrm{c}_{2}$ are the acceleration coefficients, and rand $_{1}$, rand $_{2}$ are random number between 0 and 1 .

\section{CONSTRUCTING DUAL NN COMBINED WITH PSO}

Although NNs have strong non-linear learning and representation capabilities, a traditional $\mathrm{NN}$ does not take into account the uncertainties of the weights in the network and only obtain point estimates of the weights [19]. There are many methods to reduce network complexity and increase efficiency in classification and forecasting. From [19], this paper utilizes a new cyclic NN called Dual NN, in order to classify system failure and release shedding strategy. In detail, the ANN network uses the Back Propagation (BP) algorithm to forecast power system whether load shedding or not. Another ANN uses the PSO algorithm to determine the load quantity needed to load shedding. We combine these $2 \mathrm{NNs}$ to establish forecast value and load shedding in the power system.

\section{SimUlation AND RESUltS}

The proposed method is tested on the Microgrid IEEE-25 bus diagram. This test system consists of 8 synchronous machines with excitation types AC5A and AC8B [20], 25 buses, 25 transmission lines, 9 transformers, and 11 constant impedance loads. The total load demand is $393 \mathrm{~kW}$ [21]. The diagram of the MG system is shown in Figure 2.

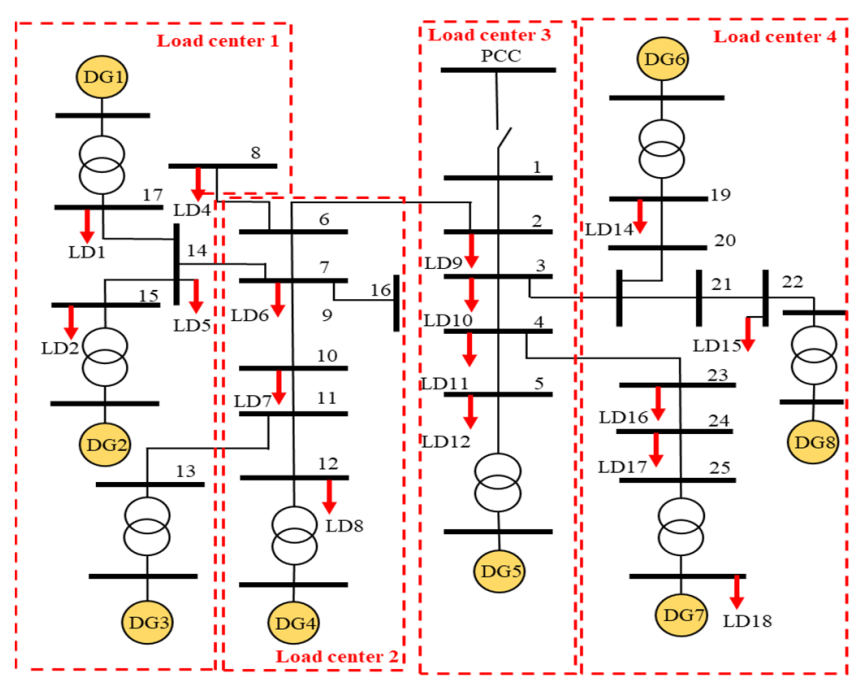

Fig. 2. Microgrid diagram of the IEEE 25 bus system.

The construction of the dataset to train the dual ANN is done with loads ranging between $50 \%$ and $100 \%$ of the load. For each load level, the power grid provides sample data with Island operation cases and generator failure cases. The process of training the dual ANN is shown in Figure 3. About load shedding cases, each different load level has a load combination and the sequence of shedding these loads which is ranked follows the priority based on the AHP algorithm. The load shedding process is conducted based on the results of load rank and is implemented until the frequency is restored to the allowable range. The summary results of load shedding strategies for different load levels are presented in Table IV.

Consider a case study at $95 \%$ load, assuming that when there is a DG5 generator problem, the load shedding combination is LS1: Load1, Load2, Load6, Load5, Load11, Load10. For a DG8 generator problem, the load shedding combination is LS3: Load1, Load2, Load6, Load5, Load11, Load10, Load7, Load4. Load shedding is conducted similarly for different generator problem cases. Recovery frequency value and frequency recovery time are within the allowable range. 


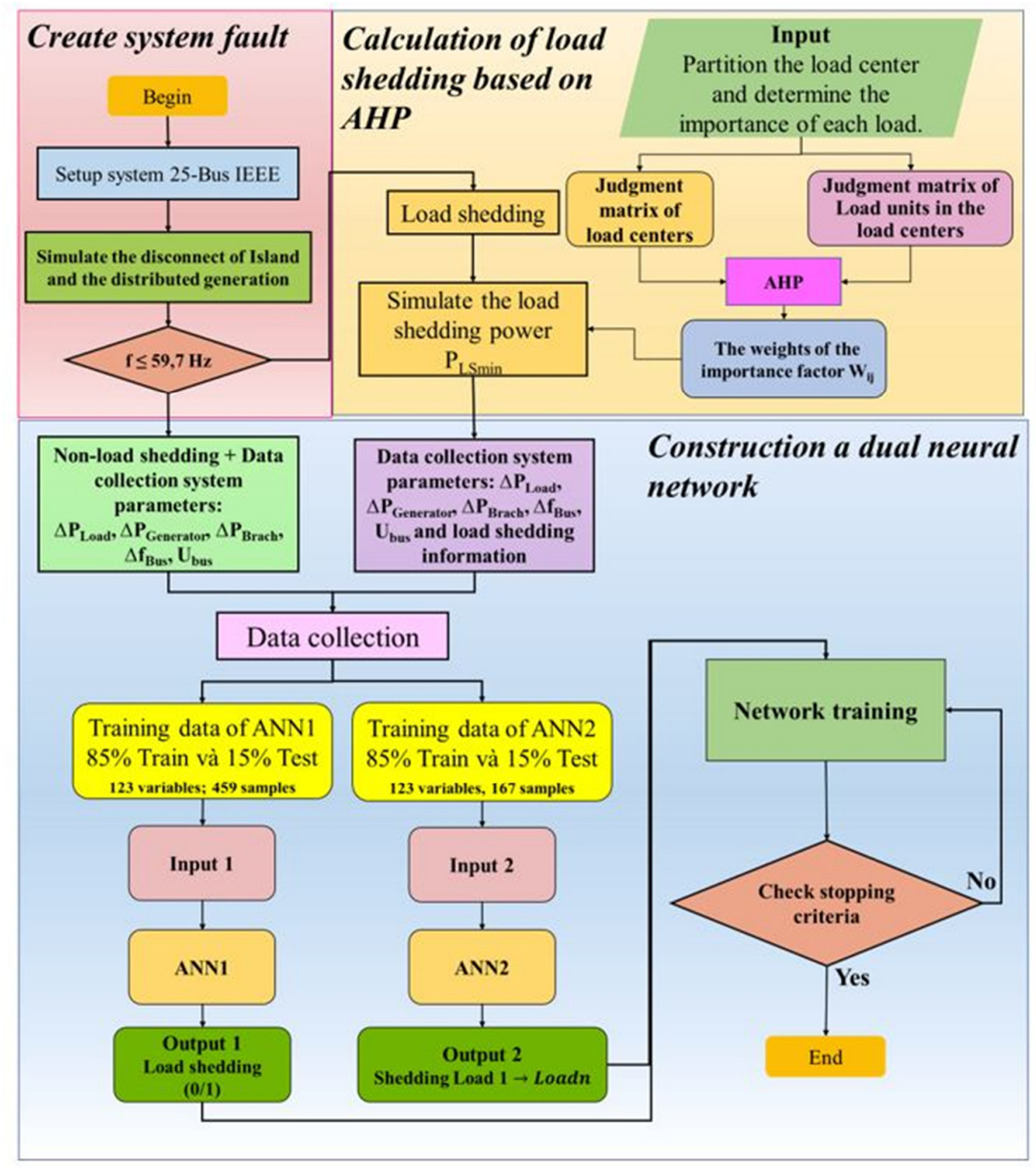

Fig. 3. Supplemental flowchart for sampling and clustering load shedding using DNN.

The results of the implementation of load shedding strategies are presented in Table $\mathrm{V}$. The comparison of recover frequency for the case with and without load shedding is shown in Figure 4. As the comparison result, when load shedding is not conducted, frequency will decrease deeply and out of the allowable range. Meanwhile, if the load shedding is performed according to the proposed method, the frequency is recovered to the allowable value ranges within $21 \mathrm{~s}$ to $30 \mathrm{~s}$.

TABLE IV. STRATEGIES FOR LOAD SHEDDING ACCORDING TO THE AHP

\begin{tabular}{|c|c|}
\hline $\begin{array}{c}\text { Load shedding } \\
\text { control strategy }\end{array}$ & Load \\
\hline $\mathrm{LS}_{1}$ & Load1, Load2, Load6, Load5, Load11, Load10 \\
\hline $\mathrm{LS}_{2}$ & Load1, Load2, Load6, Load5, Load11, Load10, Load7 \\
\hline $\mathrm{LS}_{3}$ & Load1, Load2, Load6, Load5, Load11, Load10, \\
Load7, Load4
\end{tabular}

TABLE V. FAULT STATISTICCS AND LOAD SHEDDING IMPLEMENTATION

\begin{tabular}{|c|c|c|c|c|}
\hline Case study & LS & $\begin{array}{c}\text { Recovery } \\
\text { frequency } \\
\text { (Hz) }\end{array}$ & $\begin{array}{c}\text { Amount } \\
\text { of load } \\
\text { shedding } \\
\text { power } \\
\text { (kW) }\end{array}$ & $\begin{array}{c}\text { Frequency } \\
\text { recovery } \\
\text { time (s) }\end{array}$ \\
\hline Island operation & LS2 & 50.004 & 141.25 & 23.97 \\
\hline Island operation and loss DG1 & LS3 & 50.034 & 197.84 & 22.08 \\
\hline Island operation and loss DG2 & LS4 & 50.020 & 215.83 & 20.09 \\
\hline Island operation and loss DG3 & LS3 & 50.026 & 197.84 & 28.08 \\
\hline Island operation and loss DG4 & LS3 & 50.007 & 197.84 & 25.08 \\
\hline Island operation and loss DG5 & LS3 & 50.066 & 197.84 & 30.08 \\
\hline Island operation and loss DG6 & LS3 & 50.064 & 197.84 & 25.08 \\
\hline Island operation and loss DG7 & LS3 & 50.065 & 197.84 & 23.08 \\
\hline Island operation and loss DG8 & LS3 & 50.090 & 197.84 & 21.08 \\
\hline
\end{tabular}

All the data collected through the simulation process were used for the training of the NN. The data consist of 459 training 
samples and 123 input variables including 5 system parameters, i.e. line capacity, generator, load, frequency, and each bus voltage. The output of each training sample has 15 variables consisting of: yes/no recognition status (value 1 or 0 ), load shedding, and 4 load shedding strategies. All the data are processed and 2 separated datasets are established. The first dataset includes 459 samples, with 123 input variables and 1 output variable. The first dataset used BPNN with many different improved algorithms to forecast whether load shedding should be performed or not. The second dataset includes the cases of load shedding. This dataset consists of 167 samples, with 123 input variables and 14 output variables corresponding to the state of load shedding or not. The PSOANN is utilized to predict load shedding strategies. The result of the 2 networks will be combined to show the forecast of whether load shedding or not and the suitable strategy.

(a)

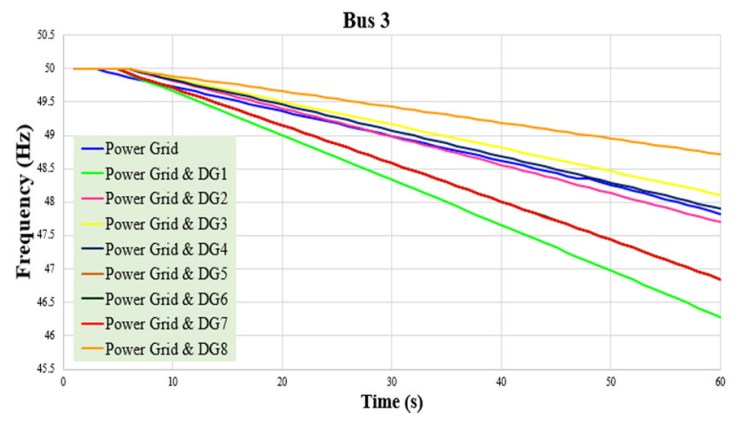

(b)

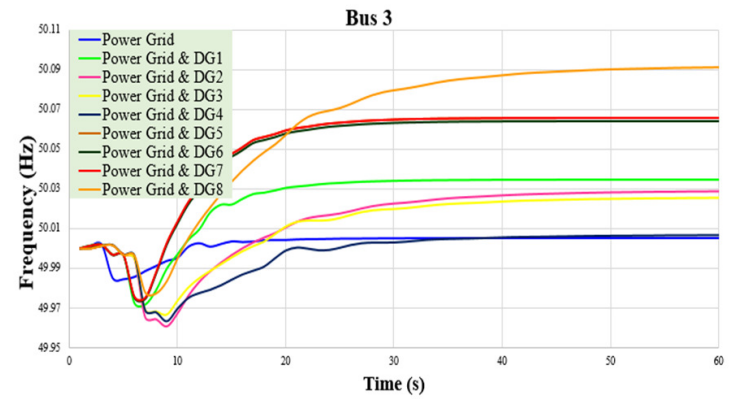

Fig. 4. Frequency characteristics at Bus 3 (a) without load shedding and (b) with load shedding based on the proposed method.

The training results in Figure 5. We can see that the result of the NN-PSO algorithm in Figure 5(a) has many advantages and is more suitable than the GA-NN with $95.59 \%$ testing and $99.74 \%$ training accuracy considering 40 input variables. The result (b) network of the improved algorithm gives good results. The Bayesian algorithm performs better with accuracy $100 \%$ in training and $96 \%$ in testing with 20 input variables. So, the BPNN will use the Bayesian algorithm to improve the network structure.

\section{DISCCUSSION}

The application of DNN combining PSO and AHP algorithms has supported the decision to load shedding quickly. That makes the power system able to keep the frequency in a steady state. The frequency of the system recovers to the allowable value. The AHP algorithm is applied to build load shedding strategies by ranking loads based on their relative importance. (a)

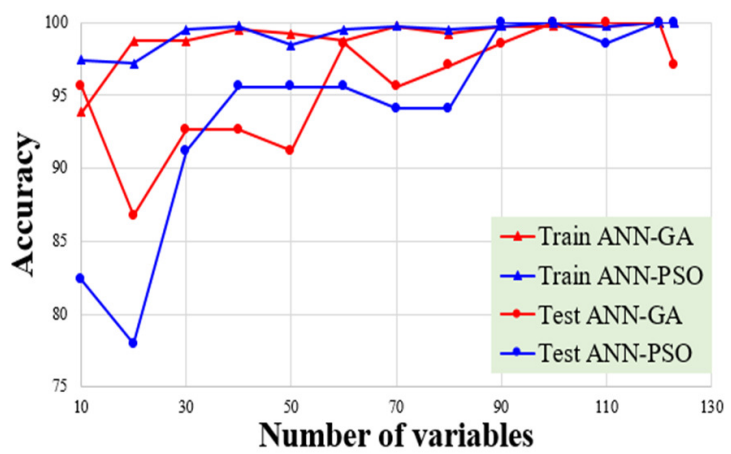

(b)

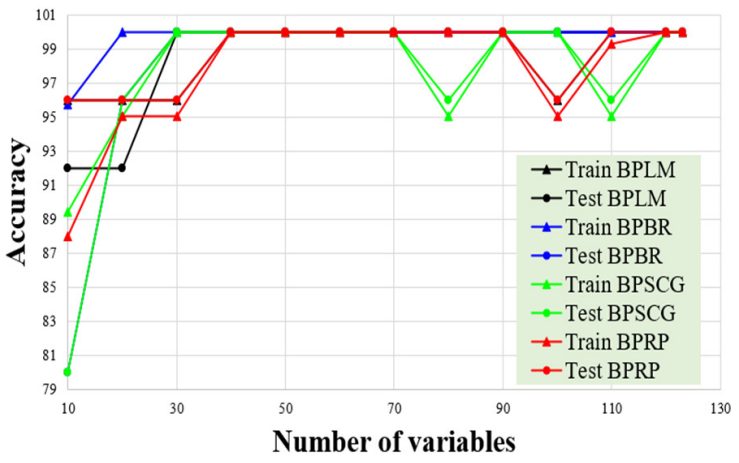

Fig. 5. Comparison chart of the training results of the proposed method with (a) Comparison of ANN-PSO and ANN-GA, (b) Comparison of the improved algorithms in BPNN network.

The proposed load shedding method used two NNs. The first (ANN1), is responsible for determining whether or not to shed the load. The second (ANN2) is responsible for determining the load shedding strategies. The training results show that using a DNN to separate the information to process, achieves training results which exhibit good performance and increase the processing speed. Meanwhile, other methods [21] perform only one task, the one of shedding the load. This shows that the proposed method is more general. However, the problem is that when the load changes continuously over time, the construction of an adaptive $\mathrm{NN}$, or a $\mathrm{NN}$ that learns and self-adjusts the network structure, will be the solutions to the above problem.

\section{CONCLUSION}

A new load shedding method based on the use of dual neural networks is proposed in this paper. A BPNN network is used to determine the severity of the failure. The result makes a yes/no decision to shed the load. The ANN-PSO network supports the proposal load shedding strategy. PSO algorithm is applied to optimize the link weights of the network, which makes accelerates the training and the accuracy of the network. The effectiveness of proposed load shedding was tested on the IEEE 25-bus 8-generator. It was shown that the proposed load shedding maintained the frequency stability in the power system.

In the future, the proposed load shedding method will be improved and along with solving the optimizing load shedding power problem, it will also reduce economic losses. 


\section{ACKNOWLEDGMENT}

This work was funded by the project grant No: T202145TĐ by Ho Chi Minh City University of Technology and Education, Vietnam.

\section{REFERENCES}

[1] N. Voropai, "Electric Power System Transformations: A Review of Main Prospects and Challenges," Energies, vol. 13, no. 21, Jan. 2020, Art. no. 5639, https://doi.org/10.3390/en13215639.

[2] C. Li et al., "Continuous Under-Frequency Load Shedding Scheme for Power System Adaptive Frequency Control," IEEE Transactions on Power Systems, vol. 35, no. 2, pp. 950-961, Mar. 2020, https://doi.org/ 10.1109/TPWRS.2019.2943150.

[3] S. S. Banijamali and T. Amraee, "Semi-Adaptive Setting of Under Frequency Load Shedding Relays Considering Credible Generation Outage Scenarios," IEEE Transactions on Power Delivery, vol. 34, no. 3, pp. 1098-1108, Jun. 2019, https://doi.org/10.1109/TPWRD.2018. 2884089.

[4] T. Amraee, M. G. Darebaghi, A. Soroudi, and A. Keane, "Probabilistic Under Frequency Load Shedding Considering RoCoF Relays of Distributed Generators," IEEE Transactions on Power Systems, vol. 33, no. 4, pp. 3587-3598, Jul. 2018, https://doi.org/10.1109/TPWRS.2017. 2787861.

[5] N. T. Le, A. T. Nguyen, T. T. Hoang, H. M. Vu Nguyen, A. H. Quyen, and B. T. T. Phan, "Distributed Load Shedding considering the Multicriteria Decision-Making Based on the Application of the Analytic Hierarchy Process," Mathematical Problems in Engineering, vol. 2021, p. e6834501, Oct. 2021, https://doi.org/10.1155/2021/6834501.

[6] M. Hasanat, M. Hasan, I. Ahmed, M. I. Chowdhury, J. Ferdous, and S. Shatabda, "An ant colony optimization algorithm for load shedding minimization in smart grids," in 2016 5th International Conference on Informatics, Electronics and Vision (ICIEV), Dhaka, Bangladesh, May 2016, pp. 176-181, https://doi.org/10.1109/ICIEV.2016.7759991.

[7] Y.-Y. Hong and C.-Y. Hsiao, "Under-frequency Load Shedding in a Standalone Power System with Wind-turbine Generators Using Fuzzy PSO," IEEE Transactions on Power Delivery, pp. 1-1, 2021, https://doi.org/10.1109/TPWRD.2021.3077668.

[8] T. Le and B. L. Nguyen Phung, "Load Shedding in Microgrids with Consideration of Voltage Quality Improvement.

[9] M. Usman, A. Amin, M. M. Azam, and H. Mokhlis, "Optimal under voltage load shedding scheme for a distribution network using EPSO algorithm," in 2018 1st International Conference on Power, Energy and Smart Grid (ICPESG), Apr. 2018, pp. 1-6, https://doi.org/10.1109/ ICPESG.2018.8384525.

[10] M. A. Zdiri, A. S. Alshammari, A. A. Alzamil, M. B. Ammar, and H. H. Abdallah, "Optimal Shedding Against Voltage Collapse Based on Genetic Algorithm," Engineering, Technology \& Applied Science Research, vol. 11, no. 5, pp. 7695-7701, Oct. 2021, https://doi.org/10. 48084/etasr.4448.

[11] T. L. Saaty, The Analytic Hierarchy Process. McGraw-Hill, New York, NY, USA, 1980

[12] H. I. Mohammed, Z. Majid, Y. B. Yamusa, M. F. M. Ariff, K. M. Idris, and N. Darwin, "Sanitary Landfill Siting Using GIS and AHP: A Case Study in Johor Bahru, Malaysia," Engineering, Technology \& Applied Science Research, vol. 9, no. 3, pp. 4100-4104, Jun. 2019, https://doi.org/10.48084/etasr.2633.

[13] H. I. Mohammed, Z. Majid, Y. B. Yamusa, M. F. M. Ariff, K. M. Idris, and N. Darwin, "Sanitary Landfill Siting Using GIS and AHP: A Case Study in Johor Bahru, Malaysia," Engineering, Technology \& Applied Science Research, vol. 9, no. 3, pp. 4100-4104, Jun. 2019, https://doi.org/10.48084/etasr.2633.

[14] H. Wang, Z. Li, L. Ma, L. Liang, G. Wu, and X. Zhang, "Prediction of CADI Chemical Composition and Heat Treatment Parameters using a BPNN Optimized with the Genetic Algorithm," IOP Conference Series: Earth and Environmental Science, vol. 233, Feb. 2019, Art. no. 052022, https://doi.org/10.1088/1755-1315/233/5/052022.
[15] Y. LeCun, Y. Bengio, and G. Hinton, "Deep learning," Nature, vol. 521, no. 7553, pp. 436-444, May 2015, https://doi.org/10.1038/nature14539.

[16] J. Zhang and S. Qu, "Optimization of Backpropagation Neural Network under the Adaptive Genetic Algorithm," Complexity, vol. 2021, Jul. 2021, Art. no. e1718234, https://doi.org/10.1155/2021/1718234.

[17] D. Wang, D. Tan, and L. Liu, "Particle swarm optimization algorithm: an overview," Soft Computing, vol. 22, no. 2, pp. 387-408, Jan. 2018, https://doi.org/10.1007/s00500-016-2474-6.

[18] R. Eberhart, Y. Shi, and J. Kennedy, Swarm Intelligence. Amsterdam, Netherlands: Elsevier Science, 2014.

[19] J. He, F. Zhuang, Y. Liu, Q. He, and F. Lin, "Bayesian dual neural networks for recommendation," Frontiers of Computer Science, vol. 13, no. 6, pp. 1255-1265, Dec. 2019, https://doi.org/10.1007/s11704-0188049-1.

[20] IEEE 421.5-2005 - IEEE Recommended Practice for Excitation System Models for Power System Stability Studies. IEEE, 2006.

[21] C. Wang, H. Yu, L. Chai, H. Liu, and B. Zhu, "Emergency Load Shedding Strategy for Microgrids Based on Dueling Deep Q-Learning," IEEE Access, vol. 9, pp. 19707-19715, 2021, https://doi.org/10.1109/ ACCESS.2021.3055401. 